\title{
GRAPPA Trainees Symposium 2019: A Report from the GRAPPA 2019 Annual Meeting
}

\author{
Catherine D. Hughes (iD, Leonieke J.J. van Mens (D), Wolf-Henning Boehncke (i), \\ and Christopher T. Ritchlin (D)
}

\begin{abstract}
The Group for Research and Assessment of Psoriasis and Psoriatic Arthritis (GRAPPA) held a trainees symposium at its 2019 annual meeting in Paris, France. Rheumatology and dermatology trainees engaged in psoriasis or psoriatic arthritis research presented their work. This report briefly reviews 5 oral presentations and 19 posters presented at the meeting. (J Rheumatol Suppl. 2020 June;96:4-10; doi:10.3899/jrheum.200120)
\end{abstract}

Key Indexing Terms: PSORIASIS TRAINEE PSORIATIC ARTHRITIS
RHEUMATOLOGIST
GRAPPA

DERMATOLOGIST
The Group for Research and Assessment of Psoriasis and Psoriatic Arthritis (GRAPPA) held this year's trainees symposium at its 2019 annual meeting in Paris, France. As in past years, rheumatology and dermatology trainees who are current GRAPPA members or who were nominated by GRAPPA members were invited to submit an abstract on recent research in psoriasis or psoriatic arthritis $(\mathrm{PsA})^{1-10}$. A total of 54 abstracts were submitted and ranked by a committee of GRAPPA members. Five trainees with the highest-scored abstracts were invited to deliver oral presentations, and 19 trainees presented posters that outlined key aspects of their research. Dr. Christopher T. Ritchlin (Rochester, New York, USA) and Dr. Wolf-Henning Boehncke (Geneva, Switzerland) co-chaired the symposium, in which GRAPPA members discussed the findings that trainees presented and

From Guy's and St. Thomas' National Health Service (NHS) Foundation Trust, London, UK; AM/University of Amsterdam, Department of Clinical Immunology and Rheumatology Amsterdam, Infection \& Immunity Institute, Amsterdam, the Netherlands; Division of Dermatology and Venereology, Geneva University Hospitals, and Department of Pathology and Immunology, University of Geneva, Geneva, Switzerland; Division of Allergy, Immunology, and Rheumatology, University of Rochester Medical Center, Rochester, New York, USA.

As part of the supplement series GRAPPA 2019, this report was reviewed internally and approved by the Guest Editors for integrity, accuracy, and consistency with scientific and ethical standards.

C.D. Hughes, Rheumatology Specialist Registrar, Guy's and St. Thomas' NHS Foundation Trust; L.J. van Mens, Resident of Rheumatology, AM/University of Amsterdam, Department of Clinical Immunology and Rheumatology Amsterdam, Infection \& Immunity Institute;

W.H. Boehncke, MD, Professor and Chair, Division of Dermatology and Venereology, Geneva University Hospitals, and Department of Pathology and Immunology, University of Geneva; C.T. Ritchlin, MD, MPH, Professor of Medicine, Division of Allergy, Immunology, and Rheumatology, University of Rochester Medical Center. C.D. Hughes and L.J. van Mens are joint first authors.

Address correspondence to Dr. C.T. Ritchlin, Professor of Medicine, Division of Allergy, Immunology, and Rheumatology, University of Rochester Medical Center, 601 Elmwood Ave., Box 695, Rochester, New York 14642,USA.E-mail:Christopher_Ritchlin@URMC.Rochester.edu suggested how trainees might further develop their current research projects.

\section{Oral Presentations}

Frequencies of $I L-17+C D 8+T$ cells and tissue-resident memory $T$ cells in early inflammatory arthritis (Catherine D. Hughes, London, UK).

Dr. Hughes sought to identify the presence and frequency of IL17+CD8+ (Tc17) T cells and/or tissue-resident memory (TRM) T cells within the joints of patients with early inflammatory arthritis (EIA). An enrichment of Tc17 cells was previously reported within PsA synovial fluid (SF). Many of these cells also have properties of TRM T cells. SF mononuclear cells were obtained from 18 EIA patients with knee effusions. Two million cells from each sample were stimulated with phorbol myristate acetate, ionomycin, and monensin and then stained for CD-3, CD-14, CD-4, CD-8, and cytokine expression [interleukin (IL)-17, interferon (IFN)- $\gamma$, and tumor necrosis factor (TNF)- $\alpha$ ]. An additional 2 million cells from 12 of these samples were stained for the presence of TRM cells (CD69+CD103+ CD8+ T cells). Samples were all acquired on BD Canto. Analysis was completed using FlowJo software. Patients were classified into disease groups based on clinical features and laboratory results. The frequency of Tc17 cells was significantly increased in the SF of patients with PsA compared to all other groups (ordinary 1-way ANOVA $\mathrm{p}<0.0001)$. Specific comparisons were seropositive rheumatoid arthritis (RA) vs PsA ( $<<0.0001)$, seronegative RA versus PsA (RA-like $p<0.0001$, oligo IA $p<0.0001$ ), and PsA versus spondyloarthritis $(\mathrm{SpA} ; \mathrm{p}<0.0001)$. There was no difference in TRM cell frequencies. This study showed that Tc17 cells are clearly found in the SF of patients with early PsA compared to other patient groups. Additionally, TRM cells are present in EIA SF. Additional work is required to establish whether these cells could function as a clinical biomarker to aid diagnosis. 
Achieving remission in PSA by early initiation of TNF inhibition: a double-blind, randomized, placebo-controlled trial of golimumab plus methotrexate versus placebo plus methotrexate (Leonieke J.J. van Mens, Amsterdam, the Netherlands).

Dr. van Mens presented a randomized, double-blinded study that assessed the effect of early initiation of the combination of methotrexate (MTX) plus a TNF inhibitor (TNFi) compared to the effect of MTX alone in patients with PsA. Fifty-one participants with active PsA were randomized to receive either treatment with golimumab (GOL) or placebo. All patients received MTX for 22 weeks. Assessed outcomes were Disease Activity Score (DAS) remission (primary outcome), joint counts, and visual analog scale (VAS) scores, as well as several other composite scores [minimal disease activity (MDA) and disease activity in PsA (DAPSA)]. Included patients had a mean disease duration of 0.5 years at baseline, as well as 5 swollen and 10 tender joints. In addition, 20 patients had a Psoriasis Area Severity Index (PASI) score of $>2.5$. Of the patients, $81 \%$ in the combination treatment group achieved the primary endpoint versus $42 \%$ in the MTX-alone group. This effect was also seen in American College of Rheumatology 20/50/70, MDA responses, and patient-reported outcome (PRO) scores. The occurrence of adverse events (AE) and serious AE was similar in both arms. The authors concluded that combination therapy of a TNFi plus MTX in patients with early PsA naive for MTX and TNFi doubled the number of patients achieving DAS remission and additional outcome measures, as well as clinical efficacy and PRO scores. This supports the concept that early initiation of a TNFi in patients with PsA favors remission.

High-dimensional flowcytometric profiling distinguishes psoriasis and PsA patients (Michelle Mulder, Nijmegen, the Netherlands).

The aims of this study were to assess whether high-dimensional flow cytometric profiling could be used to distinguish psoriasis and PsA patients, and whether it could be used to identify biomarker profiles for screening psoriasis patients who were at risk of developing PsA. The study included 25 patients with psoriasis (without coexisting PsA) and 33 biologic-naive patients with PsA. All patients with psoriasis had a Psoriasis Epidemiology Screening Tool score of $\leq 2$. Peripheral blood (PB) samples were analyzed by high-dimensional flow cytometry, which consisted of 5 different and supplementary 10-color antibody panels ( 150 immune populations). Both semi-unsupervised and unsupervised computational flow cytometry analyses were performed. In the case of semi-unsupervised analysis, the flow cytometry data were first manually analyzed, followed by multivariate principal component analysis (PCA) and unsupervised hierarchical clustering of PCA. Packages of Cytobank were used for unsupervised computational flow cytometry Flowsom and viSNE analysis. Significantly more patients with PsA used MTX compared to those with psoriasis $(51 \%$ vs $12 \%)$. Both semi-unsupervised analysis and unsupervised analysis of high-dimensional flow cytometry data of PB from psoriasis and PsA patients clearly revealed differential clustering of psoriasis versus PsA patients as indicated by PCA. This distinction between groups was independent of PASI scores and MTX use. Unsupervised computational analysis using Flowsom and viSNE showed marked differences in the immune cell subset profiles between groups. This study showed that high-dimensional flow cytometric evaluation with semi-unsupervised and unsupervised analysis can be used to differentiate psoriasis and PsA patients. The identified immune profiles might be used to aid earlier diagnosis of PsA in patients with psoriasis.

Protein engineering of the chemokine CCL2O prevents IL-23-mediated skin and joint inflammation in an IL-23 minicircle-based murine model (Zhenrui Shi, Sacramento, California, USA).

The chemokine receptor pair, CCL20/CCR6, is important for blood-to-skin trafficking of IL-17-producing T cells. CCL20LD is a novel CCL20 variant that blocks CCR6-dependent cell migration and prevents the development of psoriasis in an IL-23 intradermal injection mice model. Dr. Shi's group tested the efficacy of CCL20LD in ameliorating psoriatic inflammation with an IL-23 minicircle (MC)-based murine model with features of both psoriasis and PsA. The group showed that, in comparison to vehicle-treated mice, CCL20LD-treated mice developed significantly less skin inflammation, as measured clinically by a $50 \%$ reduction in ear thickness and psoriasis severity score. In addition, the group showed histologically a 52\% reduction in epidermal thickness with 55\% fewer Munro micro-abscesses, a 63\% reduction in infiltration of CCR6+ gamma delta low (GDL) T cells, and a 50\% reduction in mRNA levels of Th17 cytokines. Treatment with CCL20LD resulted in a 95\% reduction in IL-23 MC-induced arthritis, as measured by the arthritis severity score, and a $>75 \%$ reduction in all measured proinflammatory markers compared to the paws of their vehicle-treated counterparts. These results support the potential of engineered CCL20 molecules to disrupt CCR6-dependent inflammatory cell migration and to reduce the severity of psoriasis and PsA.

Depression and anxiety reduce probability of achieving a state of MDA in patients with PsA (Antonio Wong Lam, Toronto, Ontario, Canada).

Dr. Wong Lam questioned whether the presence of depression/anxiety leads to a lower probability of achieving a sustained MDA in patients with PsA. The presence of depression and anxiety in PsA was assessed using an existing cohort in Toronto, Ontario, Canada. Three assessments/definitions were used: definition $1, \leq 38$ on mental

Personal non-commercial use only. The Journal of Rheumatology Copyright @ 2020 . All rights reserved. 
component summary of the Medical Outcomes Study Short Form-36 questionnaire; definition $2, \leq 56$ on the mental health subscale; and definition 3, physician-reported diagnosis of depression/anxiety disorder. The primary outcome was the achievement of an MDA on at least 2 consecutive visits. In the study, $45 \%$ of the 743 included patients failed to achieve MDA. The number of patients with depression/ anxiety according to the separate definitions were group 1 : 45\%; group 2: 49\%; and group 3: $28 \%$. A reduced probability of achieving a sustained MDA was seen in all 3 groups (1: OR 0.29, 2: OR 0.33, and 3: OR 0.44). The authors concluded that the presence of anxiety and depression reduces the probability of achieving a sustained MDA in PsA. They suggest that comprehensive management of PsA should include attention to these comorbidities.

\section{Poster Presentations}

Hussein Al-Mossawi (Oxford, UK) showed that single-cell RNA sequencing reveals clonal expansion of proinflammatory synovial CD8 T cells in PsA. The authors studied the cellular landscape of PsA SF and blood at single-cell resolution, combining mass cytometry (CyTOF) with dropletencapsulated single-cell mRNA sequencing and validation by full-length transcript (Smart-seq 2) single-cell mRNA sequencing directly ex vivo. The transcriptomes and $\mathrm{T}$ cell receptor (TCR) sequences of over 78,000 cells across 6 patients were obtained, and a published algorithm was used to compare TCR amino acid sequences across individuals. CyTOF demonstrated marked expansion of memory CD8 $\mathrm{T}$ cells in the joints (compared to matched blood), with a lesser expansion of memory CD4 T cells. Single-cell RNA sequencing identified pronounced CD8 T cell clonal expansions within the joints. These clonotypes showed shared amino acid CDR3 convergence across patients, indicating that these expanded CD8 cells may recognize a common epitope. The study additionally demonstrated distinct gene expression profiles of these clonotypes, including expression of activation, tissue homing, and tissue residency markers. The chemokine receptor CXCR3 was recognized as upregulated in these synovial clones, and there was elevation of CXCL9 and CXCL10 in PsA SF. The author concluded that these data provide the strongest evidence yet that PsA may be driven by a common antigen.

Gizem Ayan (Ottawa, Ontario, Canada) assessed the effect of different joint patterns on PRO in patients with mono-oligoarthritis (1-4 joints affected) from the PsA-International Database. The study included 387 patients with a mean age of 47 years and a mean disease duration of 3.93 years. There were 194 patients $(50.1 \%)$ who had 1 affected joint, 108 (27.9\%) who had 2 affected joints, 46 (11.9\%) who had 3 affected joints, and 39 (10.1\%) who had 4 affected joints. In patients with 1 or 2 affected joints, there were higher $\mathrm{C}$-reactive protein (CRP) levels in those who had large joint involvement. Additionally, in those with 3-4 affected joints, there were higher scores on several PRO and clinical VAS scores when larger joints were involved. Overall, the author concluded that when larger joints are affected, patients score higher on PRO, VAS physician global, and CRP, indicating a worse outcome in these patients.

Claudia Camargo (Rio de Janeiro, Brazil) investigated whether PASI90-100 is the most important goal for defining the choice of immunobiological agents in moderate to severe psoriatic disease. This study presented real-life data from a cohort of patients, as well as their perspectives regarding minimal cutaneous psoriatic disease. In the study, 100 patients who were treated with anti-TNF immunobiological drugs [etanercept (ETN), infliximab (IFX), ustekinumab, or adalimumab (ADA)] from 2001 to 2017 were included. The mean drug retention rate was 47 months, with $10 \%$ of patients using the current drug for more than 96 months. Of these patients, only 13\% had a PASI between 90 and 100 , and the remainder achieved a PASI of 75 . None of the included patients were willing to change their current treatment to increase skin improvement only. The Dermatology Life Quality Index (DLQI) score for the total group was 3 , and VAS patient satisfaction was 8 . When asked in the questionnaire, $60 \%$ of patients would accept a change of drugs if it was medically advised.

Victoria Furer (Tel Aviv, Israel) assessed the immunogenicity and safety of vaccination against seasonal influenza in secukinumab-treated PsA patients. In the study, 32 patients (median age 52 yrs, 18 females) who were treated for at least 3 months with secukinumab and 17 age-matched healthy controls (median age 48.5 yrs, 6 females) were vaccinated with a 3-antigen influenza vaccine (composed of H3N3, $\mathrm{H} 1 \mathrm{~N} 1$, and B strains). Clinical and laboratory assessments were performed on the day of vaccination and 4-6 weeks later. Immunogenicity was evaluated by hemagglutination inhibition assay. The response to each antigen was defined by a 4 -fold increase in the antigen titer or by seroconversion in patients whose baseline level was $<1 / 40$. There were similar percentages of responders to all 3 strains of the virus in both groups. There was no correlation between response rates, baseline characteristics, or disease activity variables. No disease exacerbations or adverse effects were observed. The authors concluded that secukinumab treatment did not affect the humoral responses to influenza vaccination in patients with PsA.

Emma Garcia-Melchor (Glasgow, Scotland, UK) investigated the role of tendon-T cell interactions in the development of chronicity in PsA. The authors assessed the effect of damage on healthy tenocytes after stimulation with conditioned media from tendon explants or IL- $1 \beta$ by quantitative PCR (qPCR) and ELISA. T cells and tenocytes were co-cultured with or without the presence of a transwell membrane to quantify $\mathrm{T}$ cell activation (CD69 by FACS and IFN- $\gamma$ by ELISA). Changes in gene expression on tenocytes after co-culture with activated $\mathrm{T}$ cells were analyzed by qPCR

Personal non-commercial use only. The Journal of Rheumatology Copyright $(\subset) 2020$. All rights reserved. 
Results showed that tenocytes upregulated inflammatory cytokines (IL-1b, IL-6), chemokines (IL-8, CCL2, CCL5, CXCL10), and adhesion molecules (ICAM-1) in the presence of damage. An upregulation of CCL20 was observed at transcript and protein level. Conditioned media from tenocytes induced $\mathrm{T}$ cell migration. Co-culture of tenocytes and $\mathrm{T}$ cells resulted in contact-dependent activation of $\mathrm{T}$ cells that in turn had an effect on tenocytes, further upregulating the production of inflammatory mediators. Results support the role of the tendon stromal compartment in the recruitment and activation of T cells. Additionally, the production of CCL20 by tenocytes after damage could explain the preferential recruitment of Th17 or gamma delta T cells into the tendon.

Davide Geat (Verona, Italy) investigated the efficacy of secukinumab without administration of the recommended initial loading dose. This retrospective observational study included 156 adult patients with psoriasis treated with secukinumab, with 75 receiving treatment according to labeled dose with a loading period and 81 receiving treatment without initial loading dose. PASI75 responses were achieved in $60 \%$ versus $40 \%$ at Week $8(\mathrm{p}<0.01)$ and $72 \%$ versus $61 \%$ at Week $12(\mathrm{p}<0.01)$. At later timepoints the PASI75 scores were $77 \%$ and $75 \%$ at Week $16,85 \%$ and $77 \%$ at Week 32, and 79\% and 78\% at Week 48. A greater proportion of patients receiving secukinumab without the loading dose discontinued it because of inefficacy $(24.6 \%$ vs $13.3 \%$, $\mathrm{p}<0.05)$. This was particularly prevalent in those with body weight $>80 \mathrm{~kg}$. The authors concluded that when secukinumab was administered without the loading dose, a higher proportion of primary inefficacy, and inferior clinical efficacy in earlier weeks of treatment, is seen. Similar efficacy results were seen across groups after Month 3 of treatment.

Daniela Giraldo (Bogotá, Colombia) performed a cross-sectional study of patients with psoriasis from the psoriasis clinic at the Central Militar Hospital of Colombia $(n=21)$ and healthy controls $(n=11)$, with the aim of identifying subclinical findings of PsA. Disease severity was measured by a dermatologist using PASI and the Nail Psoriasis Severity Index. A radiologist performed fingernail and distal interphalangeal (DIP) joint ultrasonography (US) including Power Doppler US (PDUS) in patients and healthy controls. Patients with psoriasis had a greater frequency of erosions at the DIP joint $(6.7 \%$ vs $0 \%, \mathrm{p}<0.001)$, calcifications at the level of the extensor tendon $(63.9 \%$ vs $31.8 \%$, $\mathrm{p}<0.001)$, altered morphology of the nail plate $(20.1 \%$ vs $0 \%, \mathrm{p}<0.001)$, and higher PDUS in the nail bed $(58.2 \%$ vs $16.4 \%, p<0.001)$ and insertion of the extensor tendon (7.7\% vs $1.8 \%$, p 0.029). Patients with psoriasis showed higher rates of enthesitis compared to healthy controls (48\% vs $18 \%, p<0.001)$. Nail US could be used as a screening method for identifying patients at risk of PsA because it shows a high sensitivity, but low specificity, for detecting subclinical nail and DIP joint changes.
Clemence Gorlier (Paris, France) explored the frequency of flares in patients with PsA and assessed the validity of patient-defined flares in contrast to PsA disease activity measures. Patient- and physician-defined flares were assessed by these questions: patient-reported flare: "At this time, are you having a flare of your PsA, if this means the symptoms are worse than usual?" Physician-reported flare: "At this time, is the disease in flare (i.e., significantly worsened/more active compared to usual)?" These definitions were compared to a DAPSA-defined flare, defined by a change to a higher disease activity DAPSA category when compared to an earlier visit. Of the 222 patients analyzed, $127(57.2 \%)$ were male, the mean age was 54 years, 66\% were treated with a biologic, and the mean disease duration was 10.8 years. Disease activity was defined as moderate: $36 \%$ of patients had no current psoriasis lesions, there was a mean swollen joint count (SJC) of 1.6 (SD 6.6), and there was a mean DAPSA score of 11.5 (14). At $4.5 \pm$ 2.2 months followup, $27 \%$ of the patients reported a flare compared to $17.6 \%$ of the physicians. A flare, defined by an increase of DAPSA category, occurred in $40.1 \%$ of patients. Self-reported flares were reflected by a worsening of all clinical outcome measures in comparison to the first visit.

Alla Ishcenko (Leuven, Belgium) used the Leuven SpA biologics cohort (BioSPAR) to investigate whether the PsA patient population selected for biologics in daily practice changed over time. There were 3 defined treatment periods: period 1, 2000-2006, all patients within this period started a TNFi (TNFi available at that time were IFX, ETN, and ADA); period 2, 2006-2016, the second generation of TNFi became available (GOL and certolizumab); and period 3, 2016-2018, the third generation of biologics/small molecules became available (apremilast, ustekinumab, and secukinumab). The study included 185 white patients with baseline characteristics similar across groups. The first group had a longer disease duration and more tender joints compared to the third group, as well as having overall higher numbers of SJC, higher CRP levels, and more skin/ nail disease compared to other groups. The proportion of patients with dactylitis or enthesitis was similar across groups. The population of patients selected for treatment escalation changed significantly over time with the introduction of newer treatments. Disease duration and disease activity measures were lower in later time periods. The authors concluded that patients are now considered for treatment escalation at an earlier timepoint and with less active disease.

Carolina Isnardi (Buenos Aires, Argentina) conducted a cross-sectional study of patients with PsA according to ClASsification criteria for PsA (CASPAR) with the aim of validating the Reductive X-Ray Score for PsA (ReXSPA) in a cohort of patients with PsA. This score evaluates radiographic joint damage and juxtaarticular bone proliferation. Baseline data included clinical and laboratory

Personal non-commercial use only. The Journal of Rheumatology Copyright $(\subset) 2020$. All rights reserved. 
measures, and radiographs of hands and feet. The self-questionnaires that were administered included the Health Assessment Questionnaire, the Bath Ankylosing Spondylitis Disease Activity Index, the Bath Ankylosing Spondylitis Functional Index, the PsA Quality of Life, and the DLQI. The composite DAPSA index and Composite Psoriatic Disease Activity Index were calculated, and the presence of MDA was assessed. Two blinded readers scored 132 radiographs according to Sharp/van der Heijde modified for PsA [PsA-SvdH; median 35 (interquartile range; IQR 16.3-72.5)] and ReXSPA (median 22, IQR 7-46.3). Time to read radiographs was significantly shorter with ReXSPA than PsA-SvdH (mean $5.8 \pm 2.1$ vs $7.5 \pm 2.5$ min, $\mathrm{p}<0.0001$ ), as well as time to calculate them (mean $26.5 \pm 14.7$ vs $55.3 \pm$ $38.3 \mathrm{~s}, \mathrm{p}<0.0001$ ). Results showed that the ReXSPA index is quicker to read and calculate, and also has validity and good correlation with PsA-SvdH.

Chelsea Kelland (Sacramento, California, USA) hypothesized that MDL-1 regulates microtubule instability and bone resorption in IA. The author performed gene transfer of IL-23 in a mouse model to establish joint and skin inflammation in the presence or absence of either IL-23R or MDL-1. The overall aim was to define the contribution of IL-23R and MDL-1 in the pathogenesis of PsA. An in vivo gene transfer of IL-23 induced skin inflammation with an increased number of CD11b+LY6G+, CD11b+LY6C+, and CD11b+IL23R+ cells in skin and enhanced epidermal MDL-1+ cells. It was further demonstrated that IL-23-induced pathology was impaired in $\mathrm{IL}-23 \mathrm{R}^{\mathrm{GPP}+/+}$ (non-functional IL-23R) and MDL-1 $1^{-1}$ mice with less epidermal hyperplasia and skin (ears and dorsal skin) inflammation, compared with wild-type (WT) mice. Expression of skin inflammatory markers (K16, S100A7-9, CXCL-1, and CXCL-2), phosphorylation of nuclear factor- $\mathrm{\kappa B}$ and protein kinase $\mathrm{B}$, and neutrophil infiltration were also lower than those in WT mice. These data showed that IL-23R and MDL-1 exacerbate synovial and skin inflammation in vivo, suggesting that IL-23R and MDL-1 could be effectively targeted for the treatment of PsA.

Ari Polachek (Toronto, Ontario, Canada) presented the results from large prospective longitudinal cohorts that compared the prevalence, incidence, and types of malignancies occurring in PsA and psoriasis compared to the general population. He also assessed predictive factors for developing cancer in the psoriatic disease population. There were 2134 patients evaluated, including those with PsA included in the Toronto cohort (since 1978) and psoriasis (since 2006). Linkages were made with Cancer Care Ontario and the Ontario death registry. A total of 235 developed cancer over time (11\%). Those who developed cancer after the first visit $(\mathrm{n}=168)$ were included in this study. Standardized incidence ratios for the entire cohort were 0.89 , females 1.13 and males 0.72 , compared with the general population. The most frequently occurring malignancies were skin, breast, and hematological in PsA, and gynecological, skin, lung, and hematological in psoriasis. A predictor for malignancy was not identified, and there was no increased risk associated with biologics. In this cohort, the risk of malignancy was not increased for patients with PsA or psoriasis.

Charlotte Read (Los Angeles, California, USA) questioned whether mental health comorbidities influence patient satisfaction. The author investigated the association between mental health comorbidities and patients' perceptions of patient-provider communication quality among American adults with psoriasis. Two cross-sectional studies using the Medical Expenditure Panel Survey in adult patients with psoriasis were pooled, resulting in data on 7,356,610 adults. Psychological distress symptoms were reported as none/mild in $73 \%$ of patients, moderate in $21 \%$, and severe in $6 \%$. Of the total cohort, $91 \%$ had none or mild to moderate depressive symptoms, and the other $9 \%$ reported severe depressive symptoms. As measured by the Kessler Psychological Distress Scale, patients reporting moderate or severe psychological distress perceived a lower quality of patient-provider communication. Similar results were seen in patients reporting moderate to severe depression symptoms (according to Patient Health Questionnaire-2) and self-reported lower mental health functioning. The authors concluded that the psoriasis patients' mental health is affecting the perception of patient-provider communication.

Soumajyoti Sarkar (Davis, California, USA) isolated synovial cells (FLS) from synovial tissue biopsies of PsA, RA, and osteoarthritis ( $n=5$ for each). These cells were cultured in the presence or absence of tofacitinib with TNF- $\alpha$, IL-17A, or IL-22. Supernatants were collected on Day 5. ELISA were performed for IL-6, IL-8, and matrix metalloprotease (MMP)-3; 3-(4,5-dimethylthiazol-2-yl)-2, 5-diphenyltetrazolium bromide assay was performed for proliferation. Immunoblot studies of FLS lysates were performed to identify for STAT3/phospho-STAT3. The results showed that IL-17/IL-22/TNF- $\alpha$ induced phosphorylation of STAT3 by a 2-fold change relative to the untreated PsA FLS. PsA FLS pretreated with tofacitinib showed decreased levels of phospho-STAT3 compared to the FLS cells not treated with tofacitinib. IL-17, TNF- $\alpha$, and IL-22 also induced significant proliferation of PsA and RA FLS compared to the media and cells that were pretreated with tofacitinib. PsA and RA FLS stimulated with IL-17A, TNF- $\alpha$, or IL-22 produced significantly more IL- 6 , IL-8, and MMP-3 compared to media. These observations support a critical role for Janus kinase-STAT signaling pathways in PsA and provide mechanisms of action of tofacitinib.

David Simon (Erlangen, Germany) examined the effect of disease-modifying antirheumatic drugs (DMARD) on bone structure and strength in patients with PsA. The aim of this cross-sectional study was to investigate whether the use of MTX and biological DMARD (bDMARD) affects bone structure and biomechanical properties in patients

Personal non-commercial use only. The Journal of Rheumatology Copyright $(\subset) 2020$. All rights reserved. 
with PsA. The study included 165 patients with PsA $(79=$ no DMARD, $52=$ bDMARD, $34=$ MTX). No difference in bone variables was found between the no DMARD and the MTX group. The bDMARD group revealed significantly higher total and trabecular volumetric bone mineral density ( $p=0.001$ and $p=0.005$, respectively), as well as failure load $(p=0.012)$ and stiffness $(p=0.012)$. Despite longer disease duration, bDMARD-treated patients with PsA benefit from higher bone mass and better bone strength than those receiving MTX or no DMARD. This supports the concept of better control of PsA-related bone disease by bDMARD.

Ismihan Sunar (Ankara, Turkey) presented his work examining the association between enthesitis and disease activity, functional status, and quality of life. In this cohort study of 1130 patients from the Turkish national registry, patients who had active or past enthesitis $(553,48.9 \%)$ were compared to those without any reported enthesitis. The most common enthesitis site was Achilles insertion (39.6\% of 758 persons evaluated) followed by lumbar fifth spinous process $(36.5 \%)$. Results from this cohort showed that patients who had enthesitis had significantly greater disease activity, impaired functional status, and lower quality of life compared to those without enthesitis. Additionally, the presence of dactylitis and toenail involvement was more frequent and there was a longer diagnostic delay in the enthesitis group. The authors recommend evaluation of enthesitis in those patients with high PASI scores, skin lesions in extensor areas, and dactylitis involvement.

Ilaria Tinazzi (Ottawa, Ontario, Canada) explored the link between US and clinical assessment in PsA, as well as barriers to achieving remission. The study included 113 patients with PsA from 2 centers who had US scans of 46 joints and 12 large entheses. Joints were assessed by greyscale and PDUS using a semiquantitative score of $0-3$ per joint. The entheses were also evaluated for signs of inflammation. In this study, $37.3 \%$ of patients achieved MDA remission and 59\% achieved DAPSA remission/low disease activity. US remission was achieved in $19.4 \%$ of patients. In patients who did not achieve remission or low disease activity clinically, joints had higher PDUS scores (42.1\% for MDA-nonremission and $42.9 \%$ for DAPSA MDA/high disease activity) than the entheses (12.3\% for MDA nonremission and $14.3 \%$ for DAPSA MDA/high disease activity). There are major discrepancies between clinical assessment and US findings in PsA. US-identified joint synovitis seems to be a bigger barrier than the entheseal inflammation to achieving clinical remission.

Josephine Wright (Salt Lake City, Utah, USA) attempted to determine the length of delay in the treatment of patients with IA from time of initial diagnosis. A retrospective chart review of patients enrolled in the Veteran Health Administration with the disease classification code of IA was completed. Treatment prescriptions were assessed in the 12 months following diagnosis. The study involved 12,118 patients with IA. In these patients, a DMARD was initiated within the 12-month followup period in $60.0 \%$ of patients with RA, $64.3 \%$ of patients with PsA, and $29.6 \%$ of patients with ankylosing spondylitis (AS). A large proportion of patients started a DMARD within 30 days (41.2\%), and $50 \%$ of patients started treatment within 90 days. The percentage of patients with IA taking DMARD during the initial 12-month followup period increased from $48.8 \%$ in 2008 to $66.4 \%$ in 2015 . The authors concluded that over the years, the percentage of patients receiving DMARD in the first 12 months increased, although nontreatment remained common, particularly in patients with AS. They suggest that future studies should examine factors that influence treatment delay and effect on clinical and functional outcomes.

Xusong Wu (Sacramento, California, USA) hypothesized that short-term exposure to a Western diet (WD) induces psoriasiform dermatitis (PsD) in a CCR6-dependent manner. A WD, characterized by its high fat and simple sugar content, is thought to predispose individuals to inflammatory skin diseases such as psoriasis through the development of obesity. Dr. Wu showed that short-term feeding with a WD induces spontaneous PSD in mice without the need for concurrent obesity. After weaning, sex- and age-matched cohorts of C57BL/6 mice were fed a WD or an otherwise nutritionally matched control diet for 1 month. WD-fed mice developed a visible, spontaneous dermatitis that was characterized by mild epidermal hyperplasia with scaling, accumulation of neutrophils and IL-17A-producing GDL $\mathrm{T}$ cells, elevated gene expression of characteristic Th17 cytokines, neutrophil chemoattractants, and antimicrobial peptides. Auricular skin from WD-fed mice demonstrated an increase in Th1 markers compared to control diet-fed mice, whereas levels of Th2 markers remained comparable. In contrast to WT mice, CCR6-deficient mice fed a WD showed profoundly reduced skin inflammation, as measured by a decrease in ear thickness, infiltration of neutrophils, and mRNA levels of Th17 cytokines, suggesting that CCR6 mediates critical migration of requisite immune cells to the skin. This study showed that short-term exposure to a WD leads to CCR6-dependent, IL-17-dominant PsD, supporting the hypothesis that dietary components, rather than obesity itself, predispose mice to skin inflammation.

The GRAPPA members present at the symposium were enthusiastic about the presented abstracts, and the scientific input yielded interesting discussions and suggestions for future research. The next GRAPPA Trainees Symposium will be held in July 2020 in Brooklyn, New York, USA.

\section{REFERENCES}

1. Coates LC, Ritchlin CT. GRAPPA Trainees Symposium 2009: a report from the GRAPPA 2009 annual meeting. J Rheumatol 2011;38:526-9.

2. Ritchlin CT. GRAPPA Trainees Symposium 2010: a report from the GRAPPA 2010 annual meeting. J Rheumatol 2012;39:394-7.

Personal non-commercial use only. The Journal of Rheumatology Copyright (C) 2020. All rights reserved. 
3. Ash Z, Ritchlin CT. GRAPPA Trainees Symposium 2011: a report from the GRAPPA 2011 annual meeting. J Rheumatol 2012;39:2184-8.

4. Garg N, Touma Z, Ritchlin CT. GRAPPA Trainees Symposium 2012: a report from the GRAPPA 2012 annual meeting. J Rheumatol 2013;40:1413-8.

5. Szentpetery A, Johnson MA, Ritchlin CT. GRAPPA Trainees Symposium 2013: a report from the GRAPPA 2013 Annual Meeting. J Rheumatol 2014;41:1200-5.

6. Eder L, Tillett W, Ritchlin C. GRAPPA Trainees Symposium 2014: a report from the GRAPPA 2014 Annual Meeting. J Rheumatol 2015;42:1016-20.
7. Milliken M, Generali E, Marin J, Ritchlin CT. GRAPPA Trainees Symposium 2015: a report from the GRAPPA 2015 annual meeting. J Rheumatol 2016;43:952-8.

8. Polachek A, Stober CB, Gudbjornsson B, Ritchlin CT. GRAPPA Trainees Symposium 2016: a report from the GRAPPA 2016 annual meeting. J Rheumatol 2017;44:661-7.

9. Furer V, Manasson J, Boehncke WH, Ritchlin CT. GRAPPA Trainees Symposium 2017: a report from the GRAPPA 2017 annual meeting. J Rheumatol Suppl 2018;94:4-10.

10. Ford A, Mascia E, Boehncke W, Ritchlin C. GRAPPA Trainees Symposium 2018: a report from the GRAPPA 2018 annual meeting. J Rheumatol Suppl 2019;95:4-10. 